\title{
The effect of clouds in a galactic wind on the evolution of gas-rich dwarf galaxies
}

\author{
S. Recchi ${ }^{1,2}$ and G. Hensler ${ }^{1}$ \\ ${ }^{1}$ Institut für Astronomie der Universität Wien, Türkenschanzstrasse 17, 1180 Wien, Austria \\ e-mail: hensler@astro.univie.ac.at \\ 2 INAF - Osservatorio Astronomico di Trieste, via G.B. Tiepolo 11, 34131 Trieste, Italy \\ e-mail: recchi@oats.inaf.it
}

Received 3 July 2007 / Accepted 9 October 2007

\begin{abstract}
Context. Gas-rich dwarf galaxies are probably the closest counterparts to primeval objects we can find in the local Universe, therefore it is interesting to study their evolution in different astrophysical contexts.

Aims. We study the effects of interstellar clouds on the dynamical and chemical evolution of gas-rich dwarf galaxies. In particular, we focus on two model galaxies similar to IZw18 and NGC 1569 in comparison to models in which a smooth initial distribution of gas is assumed.

Methods. We use a 2D hydrodynamical code coupled with a series of routines able to trace the chemical products of SNeII, SNeIa and intermediate-mass stars. Clouds are simulated by adding overdense regions in the computational grid, whose locations are chosen randomly and whose density profiles match observed ones. We consider both cloud complexes put at the beginning of the simulation and a mechanism for continuous cloud formation. The clouds are inherently dynamically coupled to the diffuse gas, and they experience heat conduction from a hot surrounding gas.

Results. Due to dynamical processes and thermal evaporation, the clouds survive only a few tens of Myr. Due to the additional cooling agent, the internal energy of cloudy models is typically reduced by $20-40 \%$ compared with models of diffuse gas alone. The clouds delay the development of large-scale outflows by mass loading, therefore helping to retain a larger amount of gas inside the galaxy. However, especially in models with continuous creation of infalling clouds, their bullet effect can pierce the expanding supershell and create holes through which the superbubble can vent freshly produced metals. Moreover, assuming a pristine chemical composition for the clouds, their interaction with the superbubble dilutes the gas, reducing the metallicity. The resulting final metallicity is therefore generally lower (by $\sim 0.2-0.4 \mathrm{dex}$ ) than the one attained by diffuse models.
\end{abstract}

Key words. hydrodynamics - ISM: abundances - ISM: jets and outflows - galaxies: evolution - galaxies: individual: IZw18 galaxies: individual: NGC 1569

\section{Introduction}

Gas-rich dwarf galaxies are commonly classified into low surface-brightness dwarfs, called dwarf irregulars (dIrrs), and higher surface-brightness objects, usually called blue compact dwarf (BCD) galaxies. These classes of galaxies tend to have low metallicities, blue colors and complex and chaotic gas phases. A large fraction of these galaxies shows an ongoing star formation (SF) or at least hints that this process has been quenched in the recent past. In this case, these objects are commonly referred to as starburst galaxies and their gas consumption timescales are much shorter than the Hubble time (Kennicutt 1998), making this a transient phase of their evolution.

Owing to the energy released by stellar winds and supernovae ( $\mathrm{SNe}$ ), intense episodes of SF are also associated to the development of galactic winds or at least of large-scale outflows. The broad distinction between these two phenomena is the final fate of the outwards-directed flow of gas: galactic winds generally exceed the escape velocity while outflows do not, therefore they tend to recede towards the center of the galaxy. Clear signatures of outflows are present in NGC 1705 (Hensler et al. 1998; Heckman et al. 2001), NGC 1569 (Martin et al. 2002), NGC 3079 (Cecil et al. 2001), IZw18 (Martin 1996), NGC 3628 (Irwin \& Sofue 1996) among others. Perhaps the best examples of large-scale outflows driven by SN feedback are at large redshifts (Pettini et al. 1998, 2001). Although it is not certain, in any of the above-mentioned objects, that the metals will definitely leave the parent galaxy, indirect hints of the ubiquity of galactic winds are given by the mass-metallicity relation (Tremonti et al. 2004; Daveé et al. 2006) and effective yields (Garnett 2002).

From a theoretical point of view, the study of the evolution of gas-rich dwarf galaxies through numerical simulations has been performed by several authors in the recent past. The overall picture is that the occurrence of large-scale outflows is initially driven by the thermal pressure of a very hot, high pressurized gas and is favored by a flat distribution of the interstellar medium (ISM), which allows an easy vertical transport of material. However, since the transport of gas along the disk is very limited, outflows are not able to eject a significant fraction of the ISM, whereas the fraction of ejected metals can be very large (D'Ercole \& Brighenti 1999; MacLow \& Ferrara 1999; Recchi et al. 2001, hereafter RMD). For NGC 1569, Martin et al. (2002) derived a supersolar metal content in the galactic wind from X-ray spectra but also advocated mass-loading of it with the ISM.

Most of these studies, however, have focused on flows in homogeneous media, neglecting the multiphase nature of the ISM, although several attempts to perform multiphase 
hydrodynamical simulations have been made in the past, particularly using the so-called chemodynamical approach (Theis et al. 1992; Rieschick \& Hensler 2000; Hensler et al. 2004). The multiphase nature of the ISM, in particular its clumpiness, is observationally well established in dwarf galaxies (Cecil et al. 2001; Cannon et al. 2005; Leroy et al. 2006) and it has a solid theoretical background with the seminal work of McKee \& Ostriker (1977). According to this model, the ISM is composed by a cold neutral phase (representing the cores of molecular clouds), confined by a warm medium (with temperatures of the order of $10^{4} \mathrm{~K}$ ) and these two phases (which are in pressure equilibrium) are embedded in a hot, diluted intercloud medium (HIM), continuously produced by SN explosions and stellar winds. Sufficiently dense clouds can pierce the HIM without being swept up, so they can become embedded therein (Vieser \& Hensler 2007b). At the interface between clouds and HIM, condensation-evaporation processes establish the final fate of the cloud and its impact on the development of a galactic wind.

In two previous papers, we have studied the dynamical and chemical evolution of model galaxies similar to IZw18 (Recchi et al. 2004, hereafter Paper I) and NGC 1569 (Recchi et al. 2006, hereafter Paper II). The main results can be briefly summarized as follows:

- most of the analyzed models develop large-scale outflows. These outflows carry out of the galaxy mostly the chemical elements freshly produced during the most recent episodes of SF, with large escape fraction of metals with delayed production (like Fe and $\mathrm{N}$ );

- models with very short burst(s) of SF can cool and mix the newly formed metals in a very short timescale, whereas, when the SF is more complex, most of the metals are either directly ejected outside the galaxy through galactic winds or are confined in a too hot medium, therefore cannot contribute to the chemical enrichment of the warm ionized medium observed by emission lines from the H II gas;

- models with complex and long-lasting SF episodes reproduce the chemical composition and the abundance ratios of the above-mentioned galaxies much better than models with bursting SF.

In this paper we simulate models with structural parameters similar to IZw18 and NGC 1569 . We increase arbitrarily the gas density of some specific regions of the computational grid, in order to create a "cloudy" phase, and we address the question how and to which extent a "cloudy gas phase" alters the former results. The clouds possess a specific density profile and can be either added at the beginning of the simulation or continuously created during the evolution of the model. We then analyze the differences between the dynamical and chemical evolution of these models with the ones presented in Papers I and II. We point out that, at variance with the above-mentioned works, in this paper we will not specifically look for the best initial setups and the best assumptions in order to reproduce chemical and dynamical features of well-known objects. We will just stress the main variations produced by a clumpy initial setup. For this reason, we will also consider models which failed in Papers I and II at reproducing the observations of IZw18 and NGC 1569.

The paper is organized as follows: in Sect. 2 we briefly recall the evolution of a cloud embedded in a hot medium; in Sect. 3 we present the model and the adopted assumptions in the simulations. Results are presented in Sect. 4 (models with clouds fixed at the beginning of the simulation) and in Sect. 5 (continuous creation of clouds). Finally, a discussion and some conclusions are drawn in Sect. 6.

\section{The dynamics of clouds embedded in a hot phase}

The ubiquitous coexistence of cool to warm clouds in the hot phase of the ISM has attracted during the recent years broad attention on the interaction of such clouds with this tenuous and hot medium. The studies deal with three major effects: the influence of heat conduction, of shock fronts, and of the dynamics of the flowing hot gas, like e.g. with or without the presence of large-scale galactic outflows, on the evolution of clouds (Hartquist et al. 1986; Murray et al. 1993; Ferrara \& Shchekinov 1993; Vietri et al. 1997; Fragile et al. 2004; Marcolini et al. 2005; Vieser \& Hensler 2007b, among others). Despite the very large variety of adopted methodologies, astrophysical contexts and involved physical processes, and in spite of clearly defined problems, a broad variety but not yet uniqueness of issues can be summarized as follows:

- Moving from the idealized situation treated in analytical thermal conduction studies (e.g. Cowie \& McKee 1977) to saturated heat conduction and self-gravitating clouds can change the results from evaporation to condensation for the same state of the hot gas and the same cloud mass model (Vieser \& Hensler 2007a).

- A clumpy medium embedded in a hot flow produces mass loading, namely the seeding of material, ablated from the clouds, into the global flow. It has been demonstrated that this kind of phenomena helps in clarifying the X-ray emission in starburst galaxies like M 82 (Suchkov et al. 1996; Strickland \& Stevens 2000; Marcolini et al. 2005).

- A single cloud overrun by a shock wave can be crushed within the so-called crushing time (i.e. the time needed for the internal forward shock to cross the cloud and reach its downstream surface) and will be destroyed to smaller fragments if cooling dominates. But vice versa it also evaporates in a parameter regime with exceeding thermal conduction (Orlando et al. 2005).

- In a complex of clouds, if the cloud separation transverse to the flow is smaller than some critical value (a few times the typical cloud radius, the exact value depending on the authors), the clouds will merge into a single structure before the hot flow destroys them.

- Thermal conduction helps in stabilizing the surface of the cloud, making it less susceptible to Kelvin-Helmholtz and Rayleigh-Taylor instabilities (Orlando et al. 2005; Vieser \& Hensler 2007b). It can also generate an inward-propagating shock wave able to compress the core of the cloud.

In the present work we do not intend to simulate in great detail the interaction of a cloud or of a cloud complex with a diffuse hot medium, as made by the previously cited authors. We instead simulate galaxy models similar to well observed and studied gasrich dwarf galaxies, relaxing the hypothesis of a smooth initial gaseous distribution (as assumed in Papers I and II) and analyze how the inclusion of a clumpy medium changes the thermal and chemical evolution of the ISM. Indeed, the resolution required to properly take into consideration conductive fronts surrounding clouds is of the order of $0.1 \mathrm{pc}$ (Marcolini et al. 2005; Vieser \& Hensler 2007a,b), extremely computationally demanding in a simulation in which the large-scale evolution of the galaxy, up to distances of several kpc has to be taken into account. 


\section{Model description}

\subsection{The numerical code}

The simulations are performed by means of a $2 \mathrm{D}$ hydrocode in cylindrical coordinates based on a second-order upwind scheme (Bedogni \& D'Ercole 1986). The hydro solver is coupled with routines able to follow in detail the chemical and dynamical feedback on the galaxy as a consequence of SNeII, SNeIa and winds from intermediate-mass stars (IMS).

The chemical evolution has also an impact on the dynamics of the system, due to the assumption of a metallicity-dependent cooling function (Böhringer \& Hensler 1989). We point out that, as in the previous papers, when we plot abundances or abundance ratios produced by our models, we exclude grid points at temperature above $2 \times 10^{4} \mathrm{~K}$. This is because the gas at these temperatures would be undetectable with optical spectroscopy and its metallicity could be guessed only through X-ray analysis, whose use in dwarf galaxies is still quite uncertain (Martin et al. 2002; Ott et al. 2005). The code has been described in detail in RMD and newer implementations and improvements are reported in Papers I and II, therefore we refer the readers to these papers for technicalities.

Given the importance of thermal conduction on the shaping and final fate of clouds embedded in a hot medium, we just briefly recall the numerical method adopted to treat this physical phenomenon in our code. To solve the heat transport equation, the operator splitting method is adopted and the one-dimensional problem is solved through the Crank-Nicholson method (see also D'Ercole \& Brighenti 1999). A saturated heat flux (Cowie \& McKee 1977) is adopted if the mean free path of electrons is larger than the temperature scaleheight.

\subsection{Model parameters}

The initial configurations of our models are aimed at reproducing the main structural parameters of two very well studied gas-rich dwarf galaxies: IZw18 and NGC 1569. The initial setup is taken from our previous models and described in RMD (for IZw18) and in Paper II (for NGC 1569). As described in Sect. 3.3, the gaseous distribution is made clumpy, either perturbing the initial setup or adding clouds as the galaxy evolves, at the same rate as the SF. We also vary the initial mass function (IMF) of the stars. The models are identified through the notation XYZW, where $\mathrm{X}$ refers to the initial setup (I: setup similar to IZw18; N: setup similar to NGC 1569), Y takes into consideration whether the clouds are put in the initial setup of the galaxy or are continuously created (B: clouds present from the beginning; $\mathrm{C}$ : continuous creation of clouds). The third index refers to the adopted IMF: $S$ is for the Salpeter index $(x=1.35)$. A flatter-than-Salpeter index is also tested: A $(x=0.95)$ and a steeper index $(x=1.7)$ is denoted with $\mathrm{K}$.

Finally, also the yields from IMS are allowed to change ( $\mathrm{R}$ for Renzini \& Voli 1981; V for van den Hoek \& Groenewegen 1997; and M for Meynet \& Maeder 2002). For instance, the model called IBSR starts with a setup aimed at simulating IZw18, puts the clouds at the beginning of the simulation, assumes the yields of Renzini \& Voli (1981) for IMS and a Salpeter IMF. The yields from massive stars are taken from Woosley \& Weaver (1995), unlike for model M, where, for selfconsistency reasons, the yields of Meynet \& Maeder (2002) are assumed. In this case, the upper mass is also $60 M_{\odot}$, at variance with the $40 M_{\odot}$ value adopted for Woosley \& Weaver yields. For the SNeIa, the formulation of Matteucci \& Recchi (2001)
Table 1. Summary of model parameters.

\begin{tabular}{ccccc}
\hline \hline Model & Setup & $\begin{array}{c}\text { cloud } \\
\text { creation }\end{array}$ & IMF slope $x$ & IMS yields* \\
\hline IBSR & IZw18 & no & 1.35 & RV81 \\
IBSV & IZw18 & no & 1.35 & VG97 \\
IBAV & IZw18 & no & 0.95 & VG97 \\
ICSV & IZw18 & yes & 1.35 & VG97 \\
ICKV & IZw18 & yes & 1.70 & VG97 \\
NCSM & NGC 1569 & yes & 1.35 & MM02 \\
\hline
\end{tabular}

* RV81: Renzini \& Voli (1981); VG97: van den Hoek \& Groenewegen (1997); MM02: Meynet \& Maeder (2002).

has been adopted, with nucleosynthetic yields taken from the model W7 of Nomoto, Thielemann \& Yokoi (1984). The model parameters are summarized in Table 1.

As applied in Papers I and II, the SF is gasping (i.e. long episodes of SF are separated by short periods of quiescence). More details about the SF history of various models are given in the corresponding sections. We will be referring to diffuse models any time we will consider a model, similar to the described cloudy one, but with a homogeneous initial density distribution, described in detail either in Paper I or in Paper II.

\subsection{Cloud description}

A random generator identifies grid points in the galactic region which are the cores of the clouds. In the models in which clouds are put "ab initio" in the setup of the model (models identified with the second index "B", see Sect. 3.2), the central density is decided a priori $\left(100 \mathrm{~cm}^{-3}\right)$, and the total number of clouds is 25 . The exact number of clouds does not play a significant role; tests have been performed also with 50 or 75 clouds (reducing their masses) and this does not affect significantly the results. The probability of finding a cloud in a particular grid point is proportional to the gas density. Due to the flattened distribution of the ISM at the beginning of the simulation (see RMD or Paper II), this gives also a larger probability for the clouds to be found close to the disk. The density of the grid points outside the clouds is then reduced in order to get a consistent final gaseous mass. In models with continuous creation of clouds (identified with the second index " $C$ ") the mass is decided a priori: it is assumed that the clouds are created at the same rate as the SF rate.

As explained in Paper I, all the stars formed within an interval of time $\Delta t$ are treated as a single stellar population. After this interval of time (typically $10^{6} \mathrm{yr}$ ), a cloud is created, whose mass equals the mass of the single stellar population. Moreover, these clouds are given an initial infall velocity of $10 \mathrm{~km} \mathrm{~s}^{-1}$. For any of these models, the clouds are approximately shaped according to a radial density profile $\rho_{\mathrm{cl}} \propto R_{\mathrm{cl}}^{-1.7}$, where $R_{\mathrm{cl}}$ is the distance from the center of the cloud and the exponent of the power-law density profile is taken from observations (de Heij et al. 2002; Churchill et al. 2003; Tatematsu et al. 2004). It is important to stress that, given the dimensionality and the symmetry of our numerical code, the clouds are not spherical, but ring-like structures. The clouds are assumed to have primordial chemical composition (i.e. no metals in them) and the temperature is set to $10^{3} \mathrm{~K}$. Since this is also the minimum temperature allowed for the gas (see Papers I and II), the clouds are not in pressure equilibrium with the surrounding medium. In Sect. 4.1.1 we offer arguments demonstrating that this assumption is not unrealistic and we briefly describe models in which this assumption 

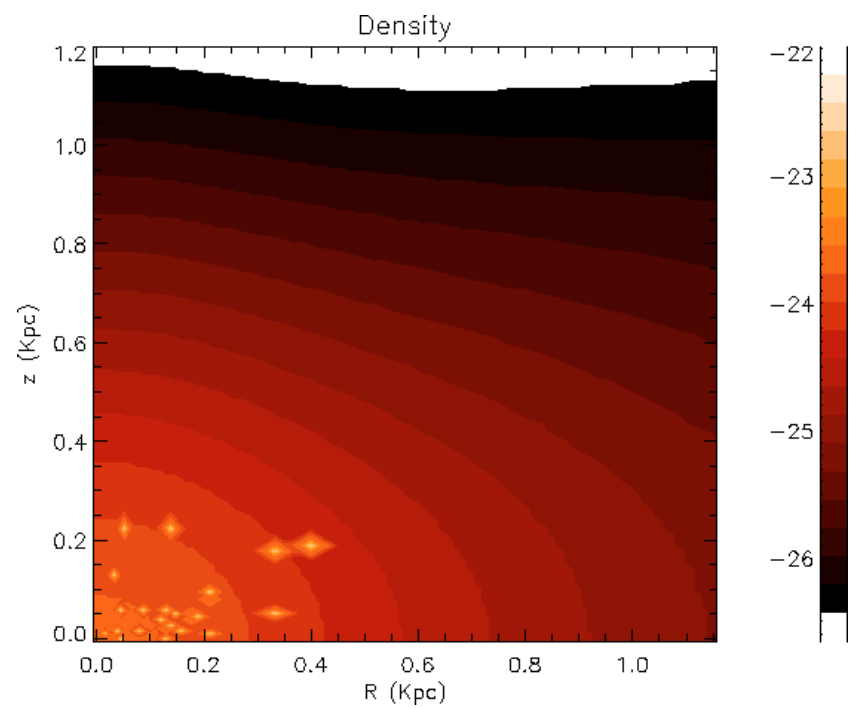

Fig. 1. Density contours for model IBSR at the beginning of the simulation. The density scale (in $\mathrm{g} \mathrm{cm}^{-3}$ ) is on the right-hand strip.

is relaxed and clouds are put in pressure equilibrium with the surrounding medium.

\section{Results of models with fixed clouds}

\subsection{Model IBSR}

\subsubsection{Dynamical evolution}

We use as a prototypical model with a fixed complex of clouds a setup similar to the model SR2 analyzed in Paper I. It has therefore the same SF history adopted therein, namely a long, moderate episode of SF lasting $270 \mathrm{Myr}$, a quiescent period of $10 \mathrm{Myr}$ and a more vigorous burst ( 5 times more intense than the first, long-lasting SF episode) lasting 5 Myr (Aloisi et al. 1999). We add randomly 25 clouds, according to the procedure described in Sect. 3.3. The initial gas distribution is shown in Fig. 1. It is worth reminding that, since the central density of the clouds is decided a priori, each cloud has a different mass, the lightest ones being close to the center. In Fig. 2 we plot the logarithmic distribution of the cloud masses. As we can see, the clouds span almost 2 orders of magnitude in mass, ranging from $\sim 10^{3.5}$ to $\sim 10^{5.5} M_{\odot}$, with a mean value of $6.26 \times 10^{4} M_{\odot}$.

The evolution of this model in the first $\sim 120 \mathrm{Myr}$ is shown in Fig. 3. This model is able to develop a large-scale outflow in a timescale of the order of $\sim 75 \mathrm{Myr}$, only slightly delayed compared to the diffuse model SR2 (for comparison, see Figs. 1 and 2 of Paper I). This model shows however a much more distorted density structure and much larger eddies and regions of thermal instabilities. As we will see better later on, the clouds are destroyed in a relatively short timescale, but nevertheless they leave an imprint on the development of the outflow, strongly influenced by the patchiness of the medium in the central region of the galaxy.

To quantify this effect, we calculate the total thermal energy of model IBSR during the first $\sim 100 \mathrm{Myr}$ and compare it with the value found for the model SR2 in Paper I. The thermal energy is calculated inside a region $R \leq 1 \mathrm{kpc}$ and $\leq 730 \mathrm{pc}$, which we have called "galactic region" in RMD and which nearly coincides with the region where stars are distributed. This comparison is shown in Fig. 4. As we can see, the total thermal energy budget is clearly affected by the presence of the clouds, leading

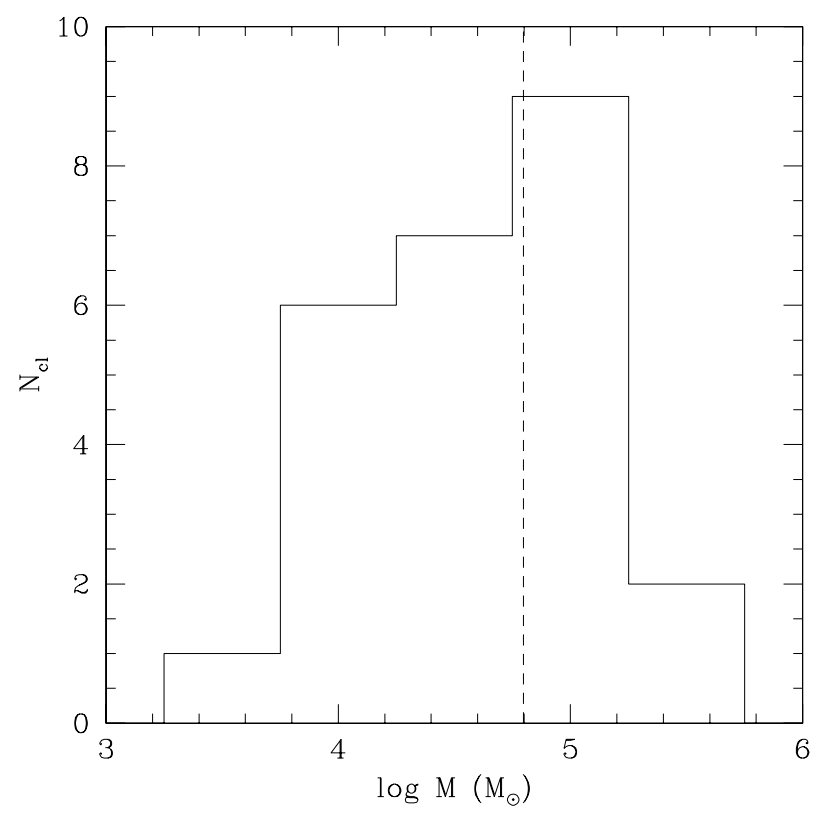

Fig. 2. Histogram of the logarithmic distribution of the cloud masses (in $M_{\odot}$ ) for the model IBSR. The dashed line represents the mean value $\left(6.26 \times 10^{4} M_{\odot}\right)$.

to a reduction of $\sim 20 \%$ of the thermal energy deposited into the system, i.e. it leads to a $\sim 20 \%$ increase of the radiative losses. Incidentally we notice that this value of the total thermal energy (corresponding to the explosion energy of just a few $\mathrm{SNe}$ ) is strongly affected, more than from the radiative cooling of the superbubble, from our assumption of a low thermalization efficiency of SNeII. An extensive discussion about this debated parameter can be found in RMD and Paper I.

In Fig. 5 we also show the average radius of the superbubble for the two above-mentioned models. The cloudy model IBSR allows at the beginning a slightly faster expansion of the superbubble. This is due to the fact that, in order to reproduce the same total mass, in a cloudy model the density of the diffuse medium has to be reduced. Moreover, the presence of clouds strongly distorts the shape of the supershell and the highly pressurized gas inside the cavity can more easily find regions of lower pressure, from which it is easy to pierce the shell and break out. This creates the tongues visible in Fig. 3. As time goes by, the larger radiative losses experienced by the model IBSR slow down the expansion of the superbubble and at $\sim 50 \mathrm{Myr}$ the average superbubble radius of the diffuse model overcomes the one of the cloudy medium.

We can better analyze the influence of clouds on the early development of galactic winds by zooming in the central region of the computational grid during the first tens of Myr. A plot of the density profiles, velocity fields and temperature profiles of the model IBSR in the first $\sim 20 \mathrm{Myr}$ is shown in Fig. 6 . We can first notice in this plot that the clouds, even if they are not encompassed in the superbubble, tend to expand. This is because they are not put in pressure equilibrium with the surrounding ISM, therefore their lifetime is relatively short (a few tens of Myr), irrespective of the presence of a surrounding HIM. We have also run models in which the clouds are put in pressure equilibrium with the surrounding ISM. This has been obtained by simply reducing the temperature of the clouds up to the value which compensate for the pressure of the ISM at the border of the cloud. This model shows the same behavior of the other models once the clouds are encompassed within the superbubble cavity, 


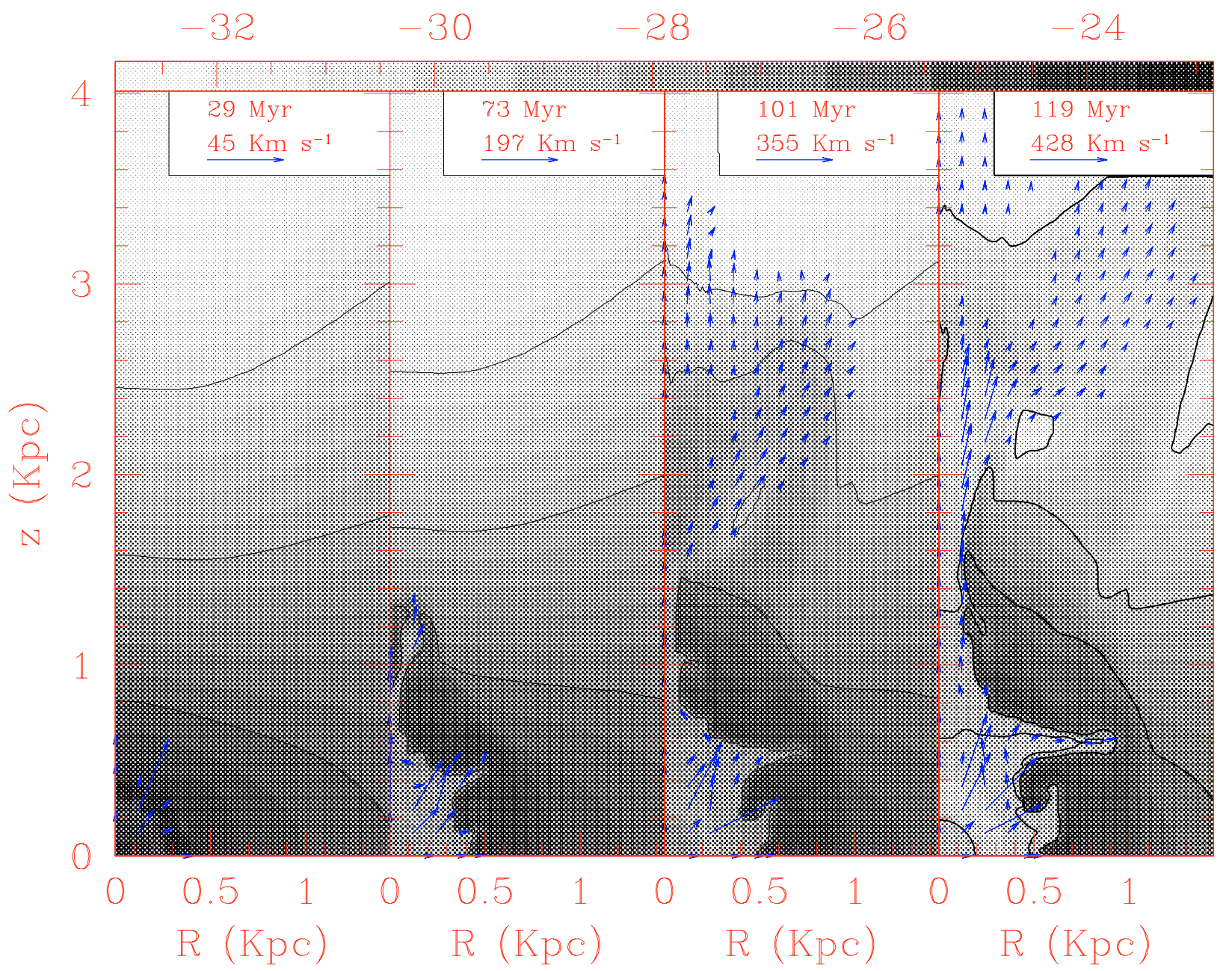

Fig. 3. Density contours and velocity fields for model IBSR at four different epochs (evolutionary times are labeled in the box on top of each panel). The logarithmic density scale (in $\mathrm{g} \mathrm{cm}^{-3}$ ) is given in the strip on top of the figure. In order to avoid confusion, velocities with values lower than 1/10 of the maximum value (indicated for each panel in the upper right box) are not drawn. This is valid also for Fig. 6.

namely they are quickly evaporated. The clouds outside the superbubble show of course a different behavior, being more stable than the previously described ones, but this does not affect the global evolution of the model. Moreover, growing evidences are accumulating, both observationally (Ballesteros-Paredes et al. 1999; Hartmann et al. 2001; Hartmann 2003) and theoretically (Elmgreen 2000; Heitsch et al. 2006) that molecular clouds are transient structures rather than well defined objects in quasiequilibrium states. Therefore they must have a relatively short lifetime, as in our simulated clouds. Given the similarities between the behavior of equilibrium and non-equilibrium clouds and due to the more complex and slow computation of equilibrium clouds, we focus from now on only on models in which the clouds are not in pressure equilibrium.

The clouds create a very patchy temperature distribution in the first 10-12 Myr but the evaporation process continues up to the moment (after $20 \mathrm{Myr}$ ) where the temperature inside the superbubble is almost uniform. We can notice once more a supershell which, owing to the interaction with clouds, strongly deviates from the spherical geometry, allowing therefore larger shears and eddies (and consequently a reduced thermal energy of the bubble), but also tongues and fingers from which it is easier to leak out of the superbubble. This is the reason why, after $\sim 100$ Myr some outflowing gas is already $\sim 3 \mathrm{kpc}$ above the galactic disk (third panel of Fig. 3) although the average superbubble radius is at this stage smaller than for the diffuse model SR2 (Fig. 5).

\subsubsection{Chemical evolution}

From a chemical point of view, the expected effect of clouds is to dilute the hot metal-rich gas through evaporation of the (metal-poor) clouds, allowing for a reduction of the metallicity without altering the abundance ratios (Köppen \& Hensler 2005). However, the reduced thermal energy and, consequently, the reduced escape fraction of metals from the galactic region (Sect. 4.1.1) should produce an increase of the metallicity of the galactic ISM. In this case, the abundance ratios are affected if the ejection efficiencies depend on the different chemical species (here we simply define ejection efficiency as the fraction of metals outside the galactic region compared to the total amount which has been synthesized). To disentangle these two competing effects, we analyze the differences in the chemical evolution between diffuse and cloudy models.

The comparison of $12+\log (\mathrm{O} / \mathrm{H}), \log (\mathrm{C} / \mathrm{O}), \log (\mathrm{N} / \mathrm{O})$ and $[\mathrm{O} / \mathrm{Fe}]$ of the warm ionized phase is presented in Fig. 7. In this plot we also show the evolution of a prototypical model of continuous creation of cloud (model ICSV, see Sect. 5.1.2). The evolution of $\alpha$-elements is not significantly altered by the presence of clouds (the difference being always around $\sim 0.1 \mathrm{dex}$ ), but we 


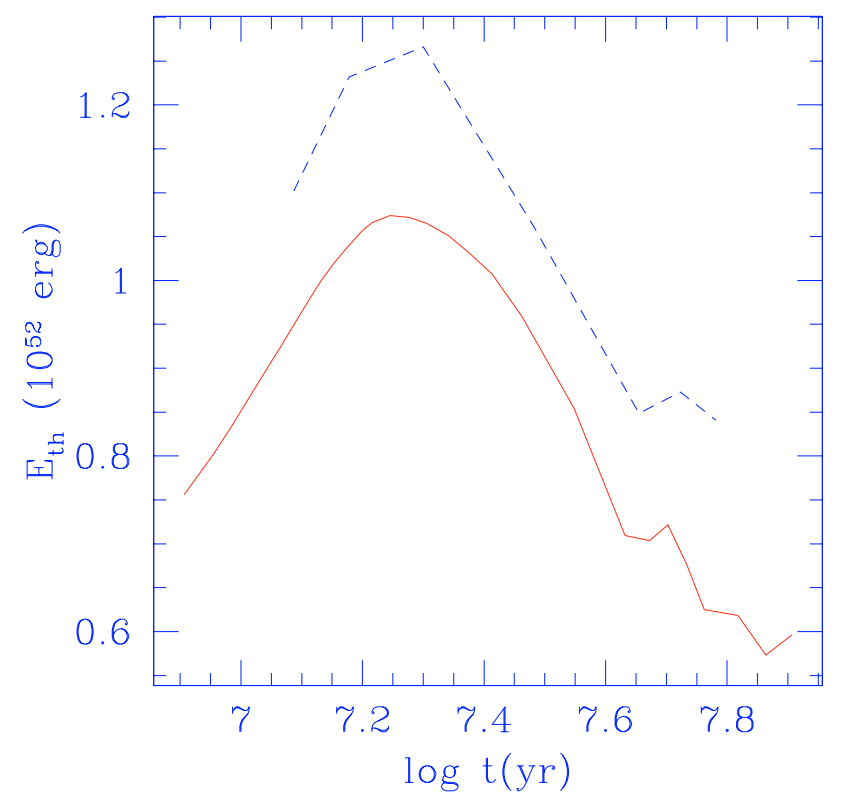

Fig. 4. Thermal energy (in units of $10^{52} \mathrm{erg}$ ) for the model IBSR (solid line) and for the reference diffuse model SR2 (Paper I) (dashed line).

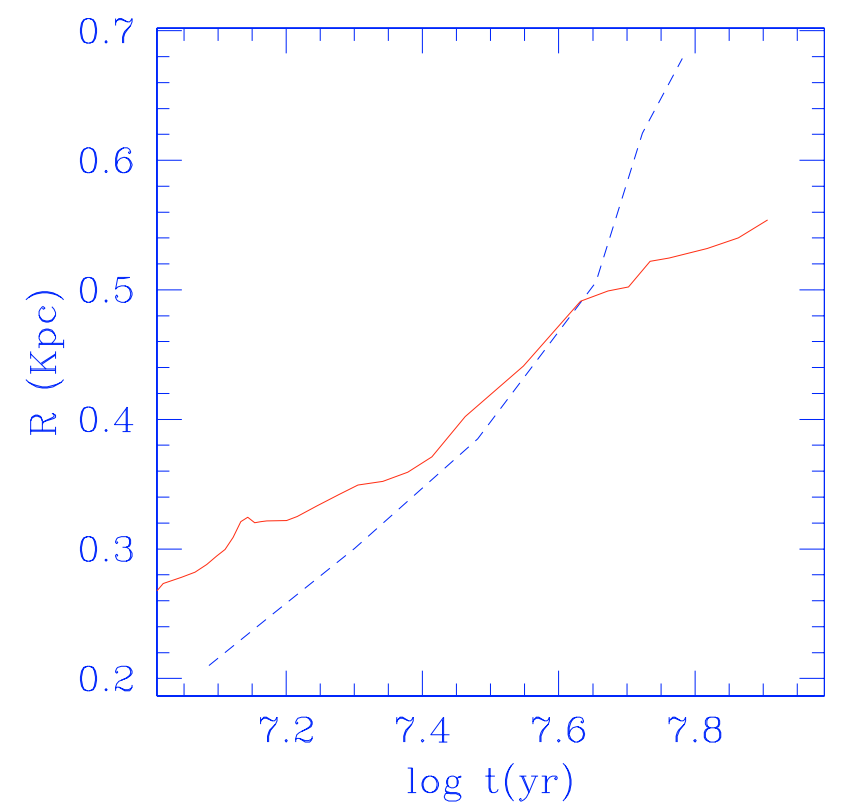

Fig. 5. Average superbubble radius (in kpc) for the model IBSR (solid line) and for the reference diffuse model SR2 (dashed line).

can notice less negligible differences (of the order of 0.2 dex) in the $\log (\mathrm{N} / \mathrm{O})$ abundance ratio. Indeed the diffuse model, due to the reduced radiative losses, attains a larger fraction of metals with temperature above $2 \times 10^{4} \mathrm{~K}$, therefore excluded by this plot. Nitrogen is mostly produced during the thermal pulsing phase by AGB stars of masses $\sim 4-7 M_{\odot}$ therefore with a delay compared to the prompt production of oxygen. Soon after its production, nitrogen is also located inside a hot cavity (carved by $\mathrm{SNe}$ ) most likely than oxygen. Moreover, as demonstrated in RMD, the ejection efficiency of nitrogen can be larger than the one of $\alpha$-elements, again favoring the decrease of N/O in the diffuse model, for which the development of a large-scale outflow is anticipated.

Incidentally, we can notice that the assumption of a cloudy medium worsens the agreement between the predicted $\log (\mathrm{N} / \mathrm{O})$

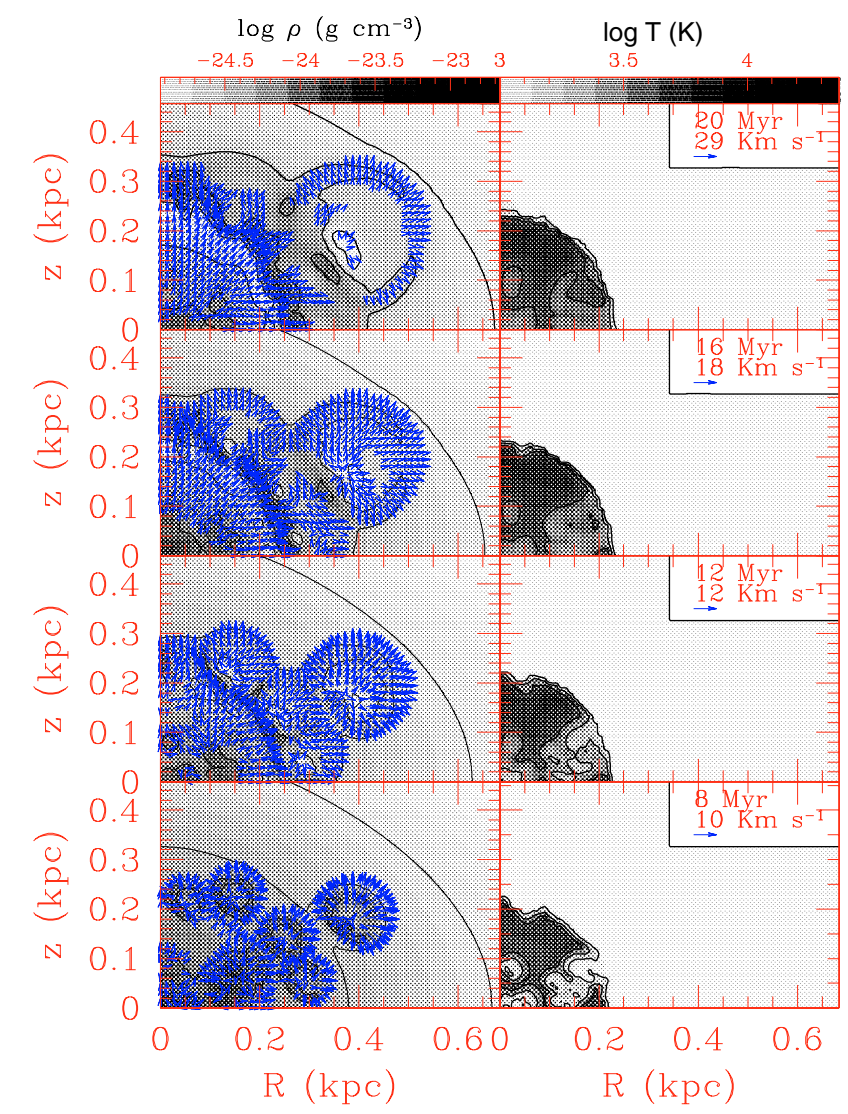

Fig. 6. Density contours and velocity fields (left panels) and temperature contours (right panels) for the central region of model IBSR at four different epochs (evolutionary times are labeled in the box on top of each right panel). Logarithmic scales are given on top of each column of panels.

and the observations. However, as we have pointed out in Paper I, the Renzini \& Voli (1981) yields tend to overestimate the nitrogen production and only the assumption of the Meynet $\&$ Maeder (2002) set of yields can reconcile the prediction of the models with the observed abundance ratios in IZw18. We stress once again that the main goal of this paper is a study of the effect of a cloud complex on the dynamical and chemical evolution of a gas-rich dwarf galaxy rather than the attempt to exactly reproduce the chemical features of specific objects.

\subsection{Model IBSV}

\subsubsection{Dynamical results}

The model IBSV differs from the model IBSR only on the assumed IMS yields (van den Hoek \& Groenewegen 1997; instead of Renzini \& Voli 1981; see Table 1). Dynamically, the only difference is therefore a variation of the cooling rates, due to our assumed metallicity-dependent cooling function. It is interesting to quantify this effect through a direct comparison of models differing solely in the adopted nucleosynthetic yields. This comparison is shown in Fig. 8 at two evolutionary times: 50 Myr (lower panels) and $100 \mathrm{Myr}$ (upper panels). Overall, the agreement between the dynamics of these two models is very good. However, the extremely non-linear character of superbubble evolution can be noticed in this plot and small differences in the energy budget of the model (due to different cooling rates) can produce significant changes in the dynamics. In particular, in model IBSR after 


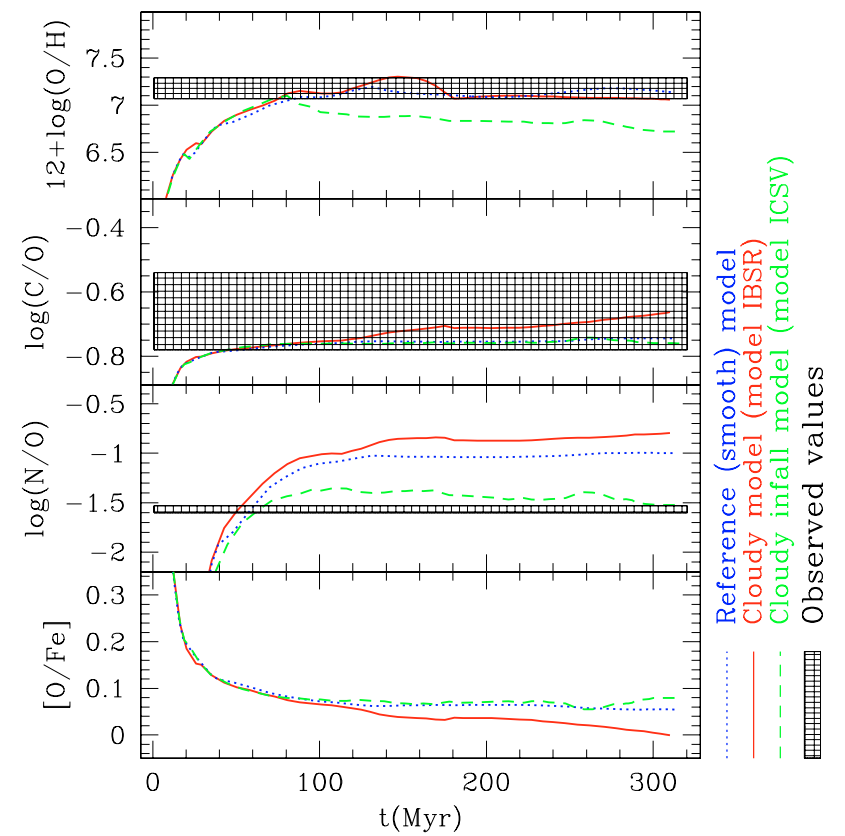

Fig. 7. Evolution of $12+\log (\mathrm{O} / \mathrm{H})$ (top panel), $\log (\mathrm{C} / \mathrm{O})$ (second panel), $\log (\mathrm{N} / \mathrm{O})$ (third panel) and $[\mathrm{O} / \mathrm{Fe}]$ (bottom panel) for a prototypical model with a fixed initial cloud complex (IBSR model) (solid line) and a prototypical model with continuous creation of cloud (ICSV model) (dashed line). These models are compared with a model of similar mass but smooth ISM distribution (dotted line). The superimposed shaded areas indicate the observed values found in literature (if available), with relative error-bars.

$100 \mathrm{Myr}$ some gas is already flowing out of the galaxy through a narrow nozzle, whereas in model IBSV the occurrence of a large-scale outflow is slightly delayed. At this point, the total mass of gas within the galaxy is $~ 9 \%$ larger in model IBSV, a small but non-negligible factor.

\subsubsection{Chemical results}

The comparison of $\log (\mathrm{C} / \mathrm{O})$ and $\log (\mathrm{N} / \mathrm{O})$ in models IBSR and IBSV is shown in Fig. 9. In this plot we do not show the evolution of oxygen because its production in IMS is negligible. Since the prescriptions for the yields from massive stars are the same (Woosley \& Weaver 1995), we do not see significant differences in the two models. The yields of van den Hoek \& Groenewegen (1997) produce substantially less carbon and nitrogen compared to Renzini \& Voli (1981) yields (Chiappini et al. 2003). The difference is particularly significant for what concerns $\mathrm{N}$, whose production is approximately halved, resulting therefore in a N/O $\sim 0.3$ dex lower than in the model IBSR. The same difference has been produced by diffuse models with different IMS yields (e.g. Recchi et al. 2002; Paper I), indicating that the different dynamics of the cloudy model do not significantly affect this behavior.

\subsection{Varying IMF: model IBAV}

As shown in Table 1, a model similar to IBSV but with flatter IMF is also considered. This models has the same SF history considered so far (i.e. the one derived from the work of Aloisi et al. 1999), but the energy injection rate is much larger. To be more precise, the total energy release is $\sim 2.5$ times larger than in IBSV. Since the gas binding energy remains the same, in spite of the larger radiative losses due to the interactions clouds-HIM, this energy release is enough to unbind all the gas initially present in the galactic region (an ellipsoid of dimensions $\sim 1000 \times 730 \mathrm{pc}$, see RMD) in $\sim 250 \mathrm{Myr}$. This complete blow-away does not happen in IBSV, where a large-scale outflow occurs in the polar direction but most of the gas close to the disk remains in the galactic region.

\section{Results of models with continuously created clouds}

As described in Sect. 3.3, in this set of models we produce a cloud each $\Delta t \mathrm{yr}$ (typical value $10^{6} \mathrm{yr}$ ), having a mass equal to the total amount of gas turned into stars in the same interval of time. In the framework of simple closed-box models of chemical evolution, this case, (infall rate equal to the SF rate) is called extreme infall (Larson 1972) and leads to the simple expression for the metallicity $Z=y_{Z}\left[1-\mathrm{e}^{-\left(\mu^{-1}-1\right)}\right]$, where $\mu$ is the gas mass fraction and $y_{Z}$ is the total yield (i.e. the ratio between the total mass in metals newly formed and the amount of mass locked up in low mass stars and remnants). The relaxation of the instantaneous recycling approximation and the inclusion of dynamical effects (winds and mixing and cooling of metals) changes this finding but Köppen \& Edmunds (1999) demonstrated that the ratio between infall and SF rate is the determining factor in the chemical evolution of galaxies. The clouds are given an infall velocity of $10 \mathrm{~km} \mathrm{~s}^{-1}$ along the polar direction, their location in the computational grid is randomly chosen and their profile is again $\rho_{\mathrm{cl}} \propto R_{\mathrm{cl}}^{-1.7}$, but in this case the central density is constrained by the total mass of the cloud and by its location rather than being constant for each cloud.

\subsection{Model ICSV}

\subsubsection{Dynamical results}

As prototype of this group of models, we use a setup similar to IBSV, the only difference being the mechanism of cloud formation. Given the assumed SF history, during the first episode the clouds have a mass of $6 \times 10^{3} M_{\odot}$, which increases to $3 \times$ $10^{4} M_{\odot}$ during the last burst. Snapshots of the evolution of this model in the first $~ 55 \mathrm{Myr}$ are shown in Fig. 10. In this figure the shocks created by the clouds in their descent to the galactic disk are quite evident, in particular in the bottom row of panels. In particular, a bow shock is created around the cloud and a reverse shock is generated downstream behind the cloud, leaving an underdensity region behind it. The structure is also highly Kelvin-Helmholtz unstable. The timescale for the growth of Kelvin-Helmholtz instabilities is approximately

$t_{\mathrm{K}-\mathrm{H}}=\frac{q^{0.5}}{k v_{\mathrm{inf}}}$,

(Chandrasekhar 1961) where $q$ is the ratio between the cloud and the intercloud densities, $v_{\text {inf }}$ is the infall velocity of the cloud (relative to the local ISM) and $k$ is the wavenumber of the unstable mode. The most unstable models are the ones with $k \sim r_{\mathrm{c}}^{-1}$ (where $r_{\mathrm{c}}$ is the radius of the cloud), leading to a $t_{\mathrm{K}-\mathrm{H}}$ between 10 and $20 \mathrm{Myr}$ with our parameters (depending on the size of the clouds, which is not constant). This is therefore also the timescale for the fragmentation of the cloud and its mixing with the local ISM. At later times, a non-negligible probability exists, given our simplified assumptions, that a cloud is created directly inside the expanding superbubble. An example is visible in the 

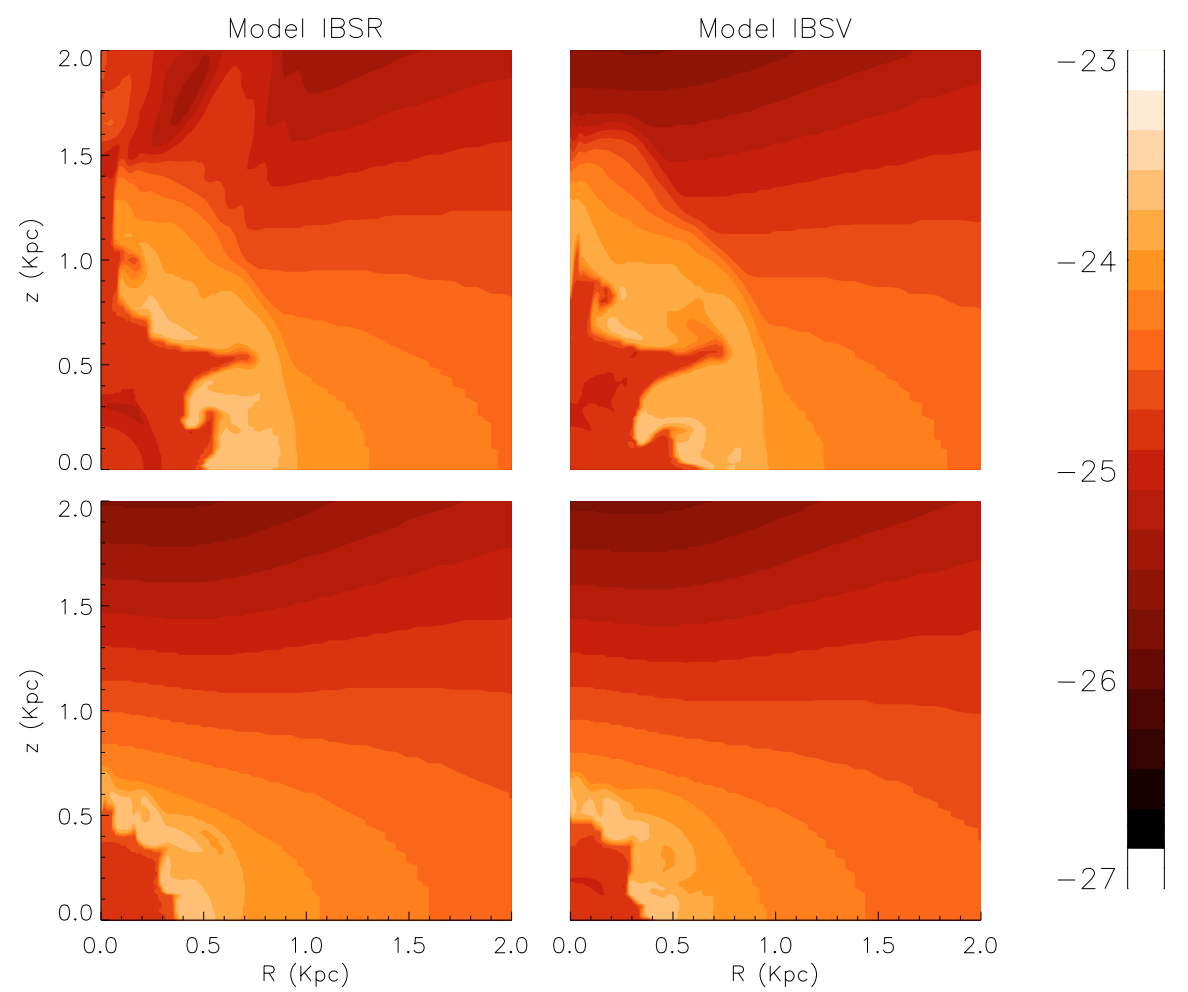

Fig. 8. Density contours for model IBSR (left panels) and model IBSV (right panels) at two evolutionary times: 50 Myr (lower panels) and $100 \mathrm{Myr}$ (upper panels). The density scale (in $\mathrm{g} \mathrm{cm}^{-3}$ ) is on the right-hand strip.

upper right panel of Fig. 10 (at $(R, z) \sim(20,200)$ pc). In this case, given the much larger density ratio $q$ (between $10^{4}$ and $10^{5}$ ), the Kelvin-Helmholtz timescale becomes larger than the time considered in our simulations (a few hundreds of Myr). The cloud is therefore ablated by the flow of gas pushed by the exploding $\mathrm{SNe}$ (creating mass loading) and evaporated by the large temperature of the cavity (few $10^{6} \mathrm{~K}$ ) in a timescale of the order of few tens of Myr. Occasionally, clouds are created close enough in space and time, such that mutual interaction between clouds manifests. In this case, the clouds form coherent structures like the ones described by Poludnenko et al. (2002) before being evaporated.

Due to the combined effect of thermal evaporation and bow shocks, the thermal energy budget is still smaller (by $\sim 15 \%$ ) than for model IBSR (plotted in Fig. 4). Owing to the larger radiative losses and to the ram pressure of the infalling clouds, a galactic wind develops after $\sim 130 \mathrm{Myr}$, delayed compared to model IBSR. However, as pointed out in Sect. 4.1.1, the infall of clouds can help in structuring and fingering the supershell, creating ways out for the hot gas. Indeed, the ram pressure of the infalling clouds is $p_{\text {ram }}=\rho_{\text {ISM }} \cdot v_{\text {inf }}^{2} \sim 10^{-11} \mathrm{erg} \mathrm{cm}^{-3}$, of the order of the ram pressure of the expanding supershell and larger than the thermal pressure of the hot cavity. Moreover, it is worth pointing out that, for this set of models, the total gaseous mass is kept constant by the assumption that the cloud creation rate should balance the SF rate (see Sect. 3.3), at variance with model IBSR, in which a fraction of gas (at a rate of $6 \times 10^{-3} M_{\odot} \mathrm{yr}^{-1}$, see Paper I) is continuously turned into stars.

\subsubsection{Chemical results}

In Paper II (Sect. 4.7) we have analyzed the effect of the infall (along the polar direction) of a very large and very massive cloud. In this case, the development of an outflow is completely hampered and all the metals freshly produced by the ongoing SF

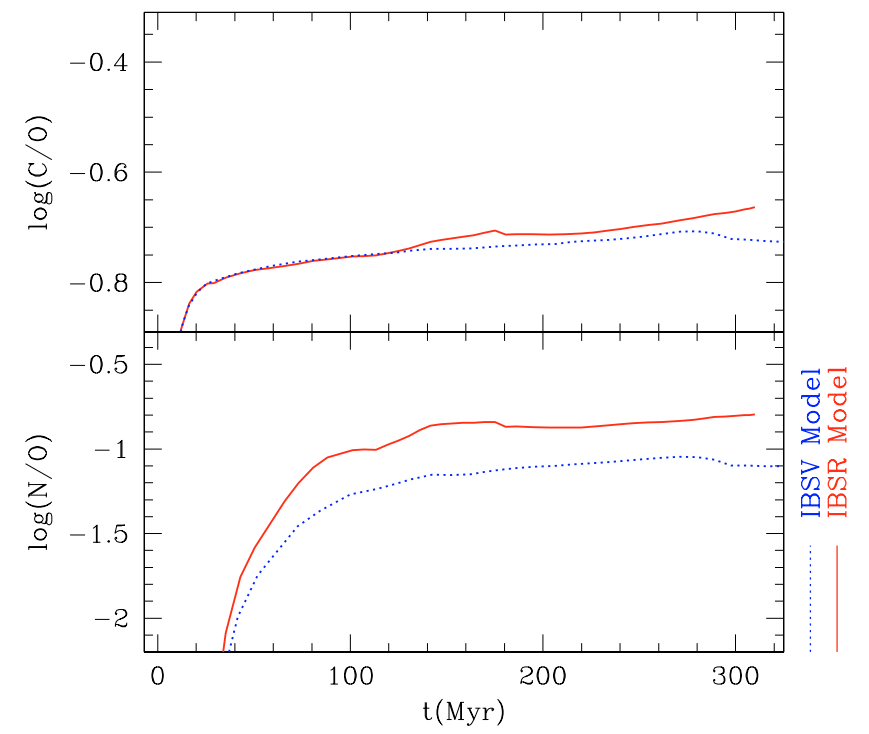

Fig. 9. Evolution of $\log (\mathrm{C} / \mathrm{O})$ (upper panel) and $\log (\mathrm{N} / \mathrm{O})$ (lower panel) for models IBSR (solid line) and IBSV (dotted line).

are trapped inside the galactic region, preventing the development of differential outflows, a natural outcome of this kind of simulations.

As we have seen, in model ICSV the "cap" effect is less significant: it helps reducing the total thermal energy able to drive a large-scale outflow, but the clouds are dissolved on a timescale of the order of a few tens of Myr. Moreover, as seen in Sect. 5.1.1, they can pierce the supershell, creating funnels for the free flow of the hot, high-pressure gas. They can therefore (slightly) delay the development of an outflow, but they cannot prevent it. 

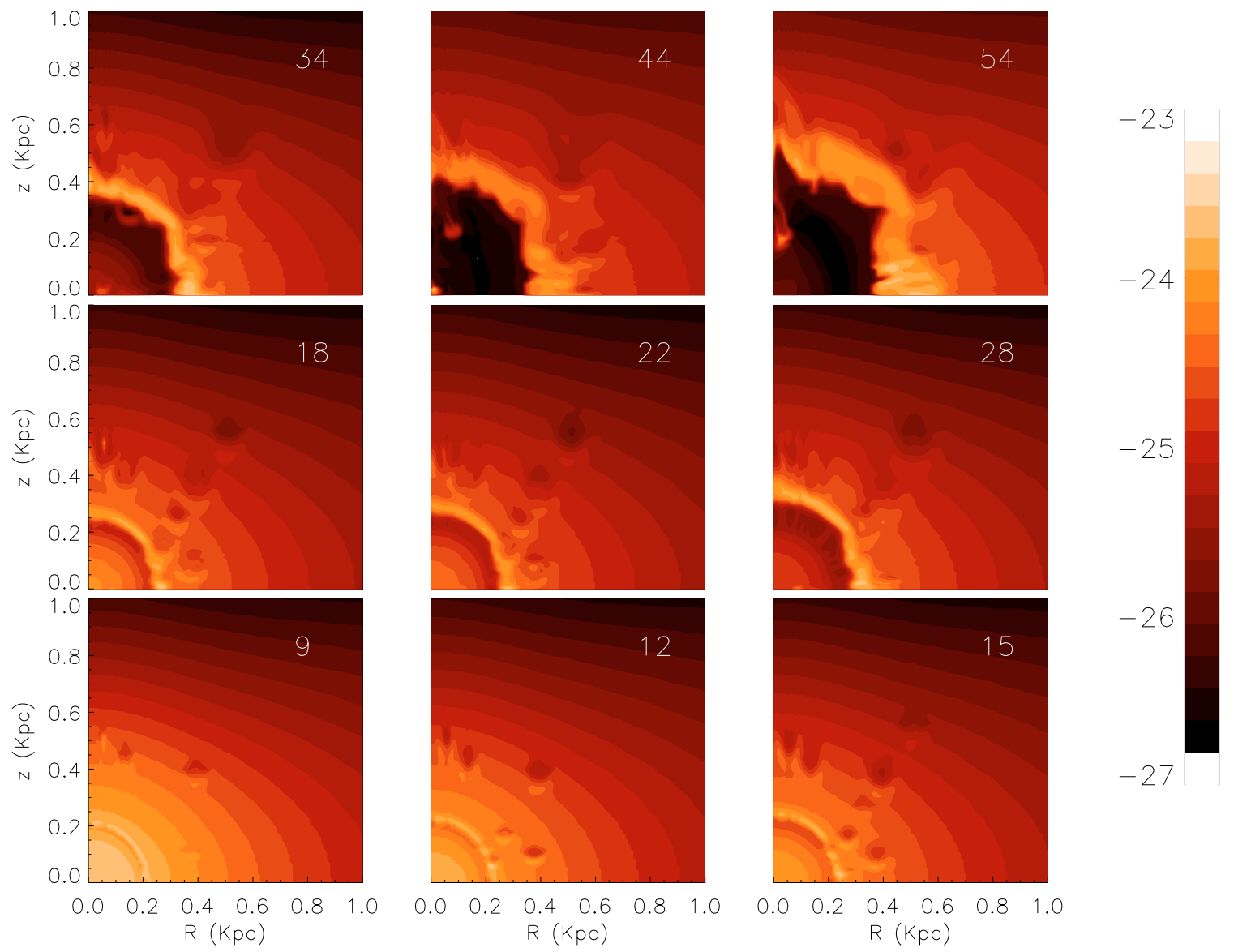

Fig. 10. Density contours for the warm gas for model ICSV at 9 evolutionary times (labeled in Myr at the top right corner of each panel). The density scale $\left(\right.$ in $\mathrm{g} \mathrm{cm}^{-3}$ ) is on the right-hand strip.

Consequently, the chemical evolution of this model is strongly affected by the dilution effect of the clouds. In particular, the process of cloud dissolution described in Sect. 5.1.1 continuously allows a mixing of the metals with pristine gas. This effect results to be, in our simulation, much larger than the "cap" effect of the infalling clouds. We can see the chemical evolution of model ICSV in Fig. 7. The final oxygen abundance is $\sim 0.3$ dex smaller that the oxygen attained by model IBSR. Even more important is the fact that, after $\sim 90 \mathrm{Myr}$, the oxygen abundance mildly but constantly decreases as function of time. This is due to the fact that the selective loss of metals has not been suppressed and that the continuous creation (and subsequent disruption) of clouds mixes the ISM with unpolluted gas. Very significant is also the effect on the N/O abundance ratio (a difference of $\sim 0.6-0.7 \mathrm{dex}$ ), but we stress once again that in this case the main reason of this difference is the choice of IMS yields. As we can see from Fig. 9, the final $\log (\mathrm{N} / \mathrm{O})$ of model IBSV is $\sim 0.3-0.4$ dex larger than model ICSV.

We also tested a model with a larger initial total mass of gas at the beginning of the simulation. The setup of this model is similar of model SV3 of Paper I (i.e. a total initial mass of $3 \times$ $10^{7} M_{\odot}$ instead of the standard value of $1.7 \times 10^{7} M_{\odot}$ ), but in it we apply the same procedure of continuous creation of clouds analyzed for model ICSV. The slightly lower density contrast parameter $q$ does not significantly affect the overall cloud-ISM interaction process and the dissolution timescale of the clumps but, due to the larger ISM pressure, the development of a largescale outflow is largely delayed. It occurs only $\sim 250 \mathrm{Myr}$ after the beginning of the SF process.

\subsection{Model ICKV}

\subsubsection{Dynamical results}

This model has the same setup and same SF history of model ICSV, the only difference being a steeper ( $x=1.7)$ IMF. Consequently, the energy return rate is much smaller than the above-considered model (about a factor $\sim 2$ ) and, since the binding energy of the gas is the same, the reduced power of the burst has deep consequences on the development of a largescale outflow. In this model, a break-out of the superbubble with consequent outflow of gas happens at around $\sim 180 \mathrm{Myr}$, but its intensity is very mild and the continuous infall of clouds is sufficient to suppress its further development and to close the funnel. The final structure (after $\sim 300 \mathrm{Myr}$ ) is an elongated ellipsoid of $\sim 700 \times 220 \mathrm{pc}$, with just some traces of gas which has managed to leak out and flows freely, mainly along the polar direction. Later on, no SF is occurring anymore, therefore the process of cloud formation is suppressed. Although the input of energy is continuous in these models (due to the contribution of SNeIa), the produced energy is not enough to break-out again, therefore the supershell tends to recede towards the center of the galaxy (see Recchi \& Hensler 2006). Only 3\% of the gas produced during the two episodes of SF leave the galactic region at the end of the simulation.

\subsubsection{Chemical results}

The chemical evolution is characterized by a strong bias towards low- and intermediate-mass stars, therefore its production 


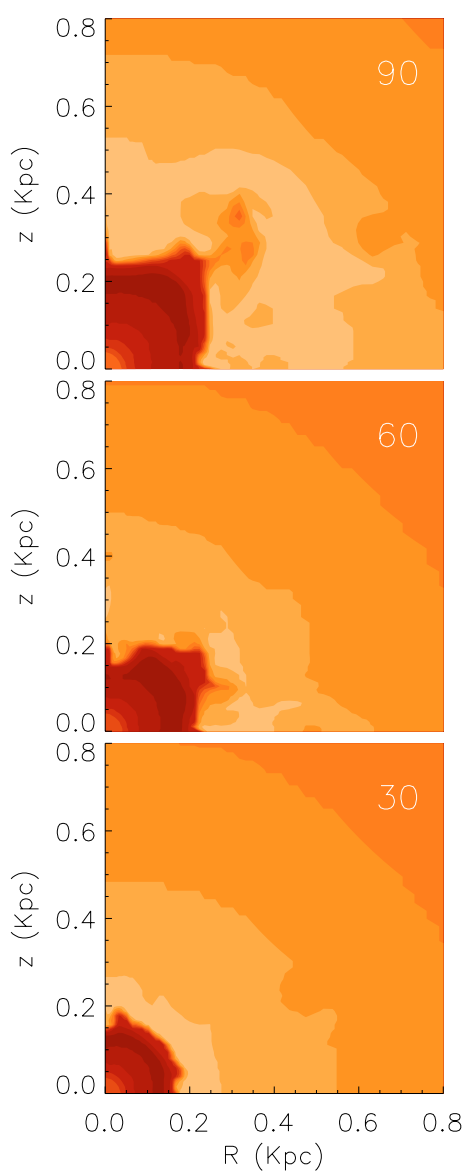

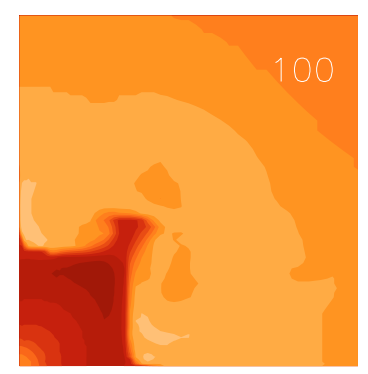
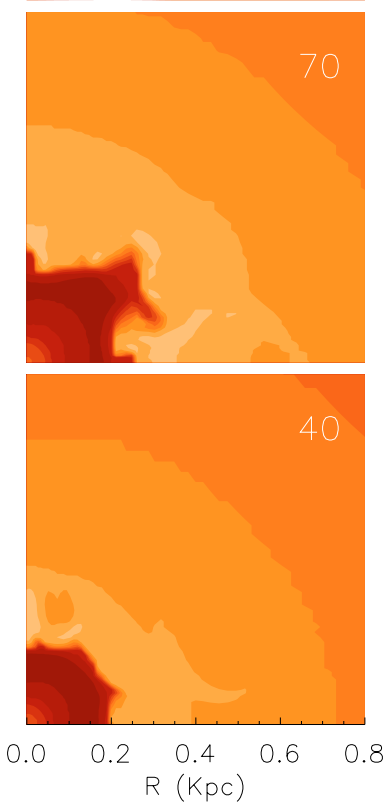

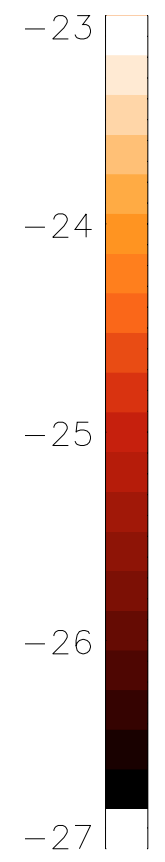

Fig. 11. Same as Fig. 10 but for model NCSM. For reference, the density contours of model NGC -5 (presented in Paper II) are shown in the upper right panel.

of $\alpha$-elements is strongly reduced. The oxygen abundance smoothly increases during the first $\sim 180$ Myr (approximately up to the break-out), then it stays almost constant. As stressed in Sect. 5.1.2, this is mainly due to the dilution effect of the clouds (as we have seen, the differential loss of metals is very limited in this model). The final abundance is $12+\log (\mathrm{O} / \mathrm{H}) \simeq$ $6.4, \sim 0.3$ dex less than model ICSV. Although in this model also the number of stars in the interval 4-7 $M_{\odot}$ (i.e. the main producers of primary nitrogen) is reduced, the final $\log (\mathrm{N} / \mathrm{O})$ is much larger ( 0.4-0.5 dex) compared to model ICSV. Finally, due to the fact that both massive and low-mass stars contribute to the final carbon abundance, the final $\log (\mathrm{C} / \mathrm{O})$ does not deviate much from the value found in model ICSV, being only $\sim 0.1$ dex larger.

\subsection{Model NCSM}

Model NCSM has an initial setup aimed at reproducing the gross characteristics of NGC 1569. For this model we assume, according to the work of Angeretti et al. (2005) three episodes of SF: a most recent one occurred between 37 and 13 Myr ago, at a rate of $0.13 M_{\odot} \mathrm{yr}^{-1}$; an intermediate episode, commencing $150 \mathrm{Myr}$ ago and finishing $40 \mathrm{Myr}$ ago, at a rate of $0.04 M_{\odot} \mathrm{yr}^{-1}$ and an older episode of SF, ending $300 \mathrm{Myr}$ ago (therefore implying $150 \mathrm{Myr}$ of inactivity between this episode and the intermediate one) and commencing $600 \mathrm{Myr}$ ago, at a rate of $0.05 M_{\odot} \mathrm{yr}^{-1}$. The setup is the same as the model NGC -5 described in Paper II, namely the total mass inside the galaxy is $1.8 \times 10^{8} M_{\odot}$, but we add continuously created clouds. At variance with the models ICSV and ICKV, the clouds are created every $5 \mathrm{Myr}$, therefore their masses are between 2 and $6.5 \times$ $10^{5} M_{\odot}$, not far from the values actually observed in the complex of H I clouds spiraling around NGC 1569 (Mühle et al. 2005) and significantly larger than the ones considered in the previous model with continuous creation of clouds. Moreover, due to the assumption made in Paper II about the nucleosynthetic prescriptions, also in this case we consider yields (from both massive and IMS) taken from Meynet \& Maeder (2002).

\subsubsection{Dynamical results}

The dynamical evolution of this model is affected by the larger masses of the clouds and by their lower creation rate. It is therefore less frequent the formation of clouds close in space and time, able therefore to mutually interact. The evolution of the model in the first $\sim 100 \mathrm{Myr}$ is shown in Fig. 11. The impact of these clouds on the development of large-scale outflows is stronger than in the above-presented cases. We can clearly see the collision of a cloud with the expanding supershell in the lower right panel of this figure. These massive clouds lead also to a stronger evaporation rate, therefore they affect significantly the energy budget of the model. The total thermal energy is $\sim 35-40 \%$ smaller than the one attained by model NGC -5 (depending on the evolutionary time). Again, as we have noticed in Sect. 5.1.1, in spite of the reduced superbubble power, the supershell-cloud interaction can create holes in the supershell from which highly pressurized gas can escape. This is visible for instance in the central right and in the upper central panel of Fig. 11. At $\sim 100 \mathrm{Myr}$ the dimension of the superbubble is only 
slightly smaller than the one attained at the same evolutionary time by model NGC -5 . This demonstrates once again that the superbubble luminosity is just one factor, in many cases not the leading one, in determining the development and the shape of large-scale outflows. Very important factors are also the density distribution and the interaction with clouds.

\subsubsection{Chemical results}

Similarly to what is seen in Sect. 5.1.2, the dilution effect of the clouds overcomes the "cap" effect, due to the delayed development of an outflow. Therefore, model NCSM shows a lower metallicity compared to model NGC -5 . In particular the oxygen abundance, after an initial phase of $\sim 250 \mathrm{Myr}$ in which it grows constantly, remains in the range $12+\log (\mathrm{O} / \mathrm{H}) \sim$ 7.6-7.7, therefore $0.3-0.4$ dex below the value attained by model NGC - 5. Similarly to the diffuse model, the last intense burst of SF has some effect on the chemical evolution of this model, resulting in an increase of $\sim 0.1-0.2$ dex. Also the $\log (\mathrm{N} / \mathrm{O})$ is reduced by $\sim 0.2-0.3$ dex compared to the corresponding diffuse model. Since we had noticed in Paper II that the results model NGC -5 matched well the observed chemical composition of NGC 1569 (taken from Kobulnicky \& Skillman 1997), we can point out that the inclusion of a cloud complex worsens the agreement between model results and observations. In order to match the observed chemical composition, one should therefore reduce the total initial mass, in order to diminish the gas fraction and therefore increase the metallicity. Playing with this parameter is allowed by the present uncertainties about the total mass of NGC 1569 (Stil \& Israel 2002; Mühle et al. 2003), but, as already pointed out in the Introduction, our main focus is not the quest for the best setup able to reproduce the chemical composition of specific objects, but the study of the effect of a cloud complex and the differences with a model in which the gaseous distribution is smooth.

\section{Discussion and conclusions}

In this paper we have computed the chemical and dynamical evolution of model galaxies, with structural parameters similar to IZw18 and NGC 1569, but in which a complex of clouds has been added, both perturbing the initial gaseous distribution and creating clouds, at a rate which equals the SF rate, and with infall velocity of $10 \mathrm{~km} \mathrm{~s}^{-1}$ along the polar direction. The main focus of our work has been the comparison of these models with those presented in previous publications, in which similar setups but a smooth distribution of gas was considered.

We have seen that the clouds are subject to a variety of disruptive phenomena like evaporation (when embedded in a hot medium), formation of shocks, development of thermal instabilities (in particular the Kelvin-Helmholtz instability) and expansion due to the larger pressure compared to the surrounding interstellar medium. The average lifetime of the clouds is therefore relatively short, depending on the cloud size (which is not constant in our simulations) but being of the order of a few tens of Myr. In spite of their transient nature, the clouds leave a significant imprint on the dynamical and chemical evolution of dwarf galaxies. The clouds, when they evaporate inside the superbubble, produce mass loading, increase the mean density of the cavity and, therefore, enhance the radiative losses (which are proportional to the square of the density). This results in a significant decrease of the total thermal energy (of the order of $\sim 20-40 \%$ compared to the diffuse models, depending on the assumptions), therefore less energy to drive the development of a large-scale outflow.

On the other hand, the relative motion of supershell and clouds, in particular when the clouds infall motion is considered, can structure, pierce and create holes and fingers in the expanding supershell. These holes destroy the spherical symmetry initially present and favor the rushing out of the highly pressurized gas contained in the cavity. Therefore, in spite of the reduced thermal energy budget, the creation of large-scale outflows is not suppressed but, in most of the explored cases, only slightly delayed. Complex structures and fingers are indeed relatively common features in galaxies showing large-scale outflows like NGC 1800 (Hunter 1996), NGC 4214 (MacKenty et al. 2000) or NGC 1705 (Heckman et al. 2001). The pressure inside the cavity is reduced compared to diffuse models, therefore in any case the total amount of ejected pristine gas is very small (smaller than in the models with smooth gas distribution) and, when averaging the size of the supershell in any direction, it turns out to be smaller than in diffuse models. But the piercing of the supershell can lead to an ejection efficiency of freshly produced metals as high as the one attained by diffuse models.

This has, of course, important consequences on the chemical evolution of these objects. Since the differential winds are not suppressed, the diminished thermal energy of these models does not imply an increase of metals inside the galactic regions. On the other hand, the dilution effect of clouds plays a dominant role in determining the final metallicity of our model galaxies. Since the clouds have primordial chemical composition, their destruction and mixing with the surrounding medium reduces the total chemical composition without altering the abundance ratios. This produces a final metallicity $\sim 0.2-0.4$ dex smaller than the corresponding diffuse models.

We have examined the effect of a different choice of the IMF slope and of the nucleosynthetic set of yields (in particular for what concerns intermediate-mass stars). Flatter-thanSalpeter IMF slopes lead to an excessive production of energy, able to unbind most of the gas before the end of the simulation. On the other hand, in models with steeper IMF the development of large-scale outflows is almost completely suppressed. Different sets of intermediate-mass stars yields affect in particular the $\log (\mathrm{N} / \mathrm{O})$ ratio. Renzini \& Voli (1981) yields tend to overestimate the primary production of nitrogen. When compared to the results of models implementing van den Hoek \& Groenewegen (1997) yields, the results differ by $\sim 0.3$ dex. Due to the assumption of a metallicity-dependent cooling function, also the dynamics is affected by the choice of the nucleosynthetic prescriptions.

Our main results can be briefly summarized as follows:

- the clouds suffer thermal instabilities, formation of shocks and evaporation, therefore their lifetimes is limited to a few tens of Myr.

- In spite of that, they are able to increase the main density of the cavity, provoking a reduction of the total thermal energy by $\sim 20-40 \%$ compared with a diffuse model.

- The interaction clouds-supershell leads to strong structuring and piercing of the shell (in particular for models with continuous creation of infalling clouds), allowing the venting out of metals in spite of the reduced thermal energy. The development of large-scale outflows is therefore generally delayed but the ejection efficiency of metals remains unchanged.

- From a chemical point of view, the effect of the clouds is to significantly reduce the total metallicity of the galaxies, without altering the abundance ratios. 
Acknowledgements. We warmly thank the referee, Peter Berczik, for his suggestions that much improved the final version of this paper. S.R. acknowledges generous financial support from the Alexander von Humboldt Foundation and Deutsche Forschungsgemeinschaft (DFG) under grant HE 1487/28-1.

\section{References}

Aloisi, A., Tosi, M., \& Greggio, L. 1999, AJ, 118, 302

Angeretti, L., Tosi, M., Greggio, L., et al. 2005, AJ, 129, 2203

Ballesteros-Paredes, J., Hartmann, L. W., \& Vázquez-Semadeni, E. 1999, ApJ, 527,285

Bedogni, R., \& D'Ercole, A. 1986, A\&A, 157, 101

Böhringer, H., \& Hensler, G. 1989, A\&A, 215, 147

Cannon, J. M., Skillman, E. D., Sembach, K. R., \& Bomans, D. J. 2005, ApJ, 618,247

Cecil, G., Bland-Hawthorn, J., Veilleux, S., \& Filippenko, A. V. 2001, ApJ, 555, 338

Chandrasekhar, S. 1961, Hydrodynamic and Hydromagnetic stability, International Series of Monographs on Physics (Oxford: Clarendon)

Chiappini, C., Romano, D., \& Matteucci, F. 2003, MNRAS, 339, 63

Churchill, C. W., Vogt, S. S., \& Charlton, J. C. 2003, AJ, 125, 98

Cowie, L. L., \& McKee, C. F. 1977, ApJ, 211, 135

Daveé, R., Finlator K., \& Oppenheimer, B. D. 2006, to appear in Proc. Chemodynamics 2006: From First Stars to Local Galaxies [arXiv:astro-ph/0608537]

de Heij, V., Braun, R., \& Burton, W. B. 2002, A\&A, 391, 67

D'Ercole, A., \& Brighenti, F. 1999, MNRAS, 309, 941

Elmegreen, B. G. 2000, ApJ, 530, 277

Ferrara, A., \& Shchekinov, Yu. 1993, ApJ, 417, 595

Fragile, P. C., Murray, S. D., Anninos, P., \& van Breugel, W. 2004, ApJ, 604, 74

Garnett, D. R. 2002, ApJ, 581, 1019

Hartmann, L. W. 2003, ApJ, 585, 398

Hartmann, L. W., Ballesteros-Paredes, L., \& Bergin, E. A. 2001, ApJ, 562, 852

Hartquist, T. W., Dyson, J. E., Pettini, M., \& Smith, L. J. 1986, MNRAS, 221, 715

Heckman, T. M., Sembach, K. R., Meurer, G. R., et al. 2001, ApJ, 554, 1021

Heitsch, F., Slyz, A. D., Devriendt, J. E. G., Hartmann, L. W., \& Burkert, A. 2006, ApJ, 648, 1052

Hensler, G., Dickow, R., Junkes, N., \& Gallagher, J. S. III 1998, ApJ, 502, L17

Hensler, G., Theis, Ch., \& Gallagher, J. S. III 2004, A\&A, 426, 25

Hunter, D. A. 1996, ApJ, 457, 671

Irwin, J. A., \& Sofue, Y. 1996, ApJ, 464, 738

Kennicutt, R. C. 1998, ApJ, 498, 541

Kobulnicky, H. A., \& Skillman, E. D. 1997, ApJ, 489, 636

Köppen, J., \& Edmunds, M. G. 1999, MNRAS, 306, 317
Köppen, J., \& Hensler, G. 2005, A\&A, 434, 531

Larson, R. B. 1972, Nature Phys. Sci., 236, 7

Leroy, A., Bolatto, A., Walter, F., \& Blitz, L. 2006, ApJ, 643, 825

MacKenty, J. W., Maíz-Apellániz, J., Pickens, C. E., Norman, C. A., \& Walborn, N. R. 2000, AJ, 120, 3007

Mac Low, M.-M., \& Ferrara, A. 1999, ApJ, 513, 142

Marcolini, A., Strickland, D. K., D’Ercole, A., Heckman, T. M., \& Hoopes, C. G. 2005, MNRAS, 362, 626

Martin, C. L. 1996, ApJ, 465, 680

Martin, C. L., Kobulnicky, H. A., \& Heckman, T. M. 2002, ApJ, 574, 663

Matteucci, F., \& Recchi, S. 2001, ApJ, 558, 351

McKee, C. F., \& Ostriker, J. P. 1977, ApJ, 218, 148

Meynet, G., \& Maeder, A. 2002, A\&A, 390, 561

Mühle, S., Klein, U., Wilcots, E. M., \& Hüttermeister, S. 2003, ANS, 324, 40

Mühle, S., Klein, U., Wilcots, E. M., \& Hüttermeister, S. 2005, AJ, 130, 524

Murray, S. D., White, S. D. M., Blondin, J. M., \& Lin, D. N. C. 1993, ApJ, 407,

Nomoto, K., Thielemann, F.-K., \& Yokoi, K. 1984, ApJ, 286, 644

Ott, J., Walter, F., \& Brinks, E. 2005, MNRAS, 358, 1423

Pettini, M., Kellogg, M., Steidel, C. C., et al. 1998, ApJ, 508, 539

Pettini, M., Shapley, A. E., Steidel, C. C., et al. 2001, ApJ, 554, 981

Poludnenko, A. Y., Frank, A., \& Blackman, E. G. 2002, ApJ, 576, 832

Orlando, S., Peres, G., Reale, F., et al. 2005, A\&A, 444, 505

Recchi, S., \& Hensler, G. 2006, A\&A, 445, L39

Recchi, S., Matteucci, F., \& D'Ercole, A. 2001, MNRAS, 322, 800 (RMD)

Recchi, S., Matteucci, F., D’Ercole, A., \& Tosi, M. 2002, A\&A, 384, 799

Recchi, S., Matteucci, F., D’Ercole, A., \& Tosi, M. 2004, A\&A, 426, 37 (Paper I)

Recchi, S., Hensler, G., Angeretti, L., \& Matteucci, F. 2006, A\&A, 445, 875 (Paper II)

Renzini, A., \& Voli, M. 1981, A\&A, 94, 175

Rieschick, A., \& Hensler, G. 2000, in Cosmic Evolution and Galaxy Formation: Structure, Interactions and Feedback, ed. J. Franco, E. Terlevich, O. López-Cruz, \& I. Arextaga (San Francisco: ASP), ASP Conf. Ser., 215, 130

Stil, J. M., \& Israel, F. P. 2002, A\&A, 392, 473

Strickland, D. K., \& Stevens, I. R. 2000, MNRAS, 314, 511

Suchkov, A. A., Berman, V. G., Heckman, T. M., \& Balsara, D. S. 1996, ApJ, 463,528

Tatematsu, K., Umemoto, T., Kandori, R., \& Sekimoto, Y. 2004, ApJ, 606, 333

Theis, Ch., Burkert, A., \& Hensler, G. 1992, A\&A, 265, 465

Tremonti, C. A., Heckman, T. M., Kauffmann, G., et al. 2004, ApJ, 613, 898

van den Hoek, L. B., \& Groenewegen, M. A. T. 1997, A\&AS, 123, 305

Vieser, W., \& Hensler, G. 2007a, A\&A, 472, 141

Vieser, W., \& Hensler, G. 2007b, A\&A, 475, 251

Vietri, M., Ferrara, A., \& Miniati, F. 1997, ApJ, 483, 262

Woosley, S. E., \& Weaver, T. A. 1995, ApJS, 101, 181 